\title{
Errata for Quantitative Robust Uncertainty Principles and Optimally Sparse Decompositions (DOI: 10.1007/s10208-004-0162-x)
}

Emmanuel J. Candès and Justin Romberg

Applied and Computational Mathematics

California Institute of Technology

Pasadena, CA 91125, USA

In the proof of Theorem 4.1, $\Phi=\left(\begin{array}{ll}I & F^{*}\end{array}\right)$ is the dictionary constructed by concatenating the Dirac and Fourier orthobases, and $\Phi_{\Gamma}, \Phi_{\Gamma^{\prime}}$ are subdictionaries constructed by extracting columns from $\Phi$ corresponding to the index sets $\Gamma, \Gamma^{\prime}$. The assertion is made that if $|\Gamma|=\left|\Gamma^{\prime}\right|$, and if both $\Phi_{\Gamma}, \Phi_{\Gamma^{\prime}}$ are full rank, then it must follow that Range $\left(\Phi_{\Gamma \backslash \Gamma^{\prime}}\right)=\operatorname{Range}\left(\Phi_{\Gamma^{\prime} \backslash \Gamma}\right)$. This is true if $\Phi_{\Gamma}$ and $\Phi_{\Gamma^{\prime}}$ are both orthogonal matrices, but is false in general (including the context of the Theorem).

A correct proof of Theorem 4.1 requires a different tack. The statement is the same, except with a very minor change in the constant. We will also not require Lemma 4.2.

Theorem 4.1. Let $f=\Phi \alpha$ be a signal of length $N \geq 512$ with support set $\Gamma=T \cup \Omega$ sampled uniformly at random with

$$
|T|+|\Omega| \leq \frac{.2681 N}{\sqrt{(\beta+1) \log N}},
$$

and with coefficients $\alpha$ sampled as in Section 2 . Then the solution to $\left(P_{0}\right)$ is unique and equal to $\alpha$ with probability at least $1-O\left((\log N)^{1 / 2} \cdot N^{-\beta}\right)$. 
Proof. Theorem 3.1 is easily generalized so that if $\Gamma$ is chosen uniformly at random with

$$
|T|+|\Omega| \leq \frac{.5583 q N}{\sqrt{(\beta+1) \log N}}
$$

for any $0<q \leq 1 / 2$, then

$$
\left\|F_{\Omega T}^{*} F_{\Omega T}\right\| \leq q
$$

with probability $1-O\left((\log N)^{1 / 2} \cdot N^{-\beta}\right)$. We will show that taking $q$ just less than $1 / 2(q \approx .4802)$ will guarantee (with probability 1$)$ that a random coefficient sequence on a $\Gamma$ which satisfies (4.1) can be recovered by solving $\left(P_{0}\right)$.

Given a $\Gamma$ obeying (4.1), the (continuous) probability distribution on the $\{\alpha(\gamma), \gamma \in$ $\Gamma\}$ induces a continuous probability distribution on Range $\left(\Phi_{\Gamma}\right)$. We will show that for every $\Gamma^{\prime} \neq \Gamma$ with $\left|\Gamma^{\prime}\right| \leq|\Gamma|$

$$
\operatorname{Range}\left(\Phi_{\Gamma^{\prime}}\right) \neq \operatorname{Range}\left(\Phi_{\Gamma}\right)
$$

As such, the set of signals in Range $\left(\Phi_{\Gamma}\right)$ that have expansions on alternate supports $\Gamma^{\prime}$ that are at least as sparse as their expansions on $\Gamma$ is at most a finite union of subspaces of dimension strictly smaller than $|\Gamma|$. This set has measure zero as a subset of Range $\left(\Phi_{\Gamma}\right)$, and hence the probability of observing such a signal is zero.

Consider any $\Gamma^{\prime}=T^{\prime} \cup \Omega^{\prime}$ different than $\Gamma$ with $\left|\Gamma^{\prime}\right| \leq|\Gamma|$. The range of $\Phi_{\Gamma}$ will equal the range of $\Phi_{\Gamma^{\prime}}$ only if each column $\varphi_{\gamma}$ for $\gamma \in \Gamma^{\prime} \backslash \Gamma$ is in the range of $\Phi_{\Gamma}$. Without loss of generality, suppose $T^{\prime} \backslash T \neq \emptyset$ (the same argument, with the roles of time and frequency reversed, also applies to the case where $\Omega^{\prime} \backslash \Omega \neq \emptyset$ ). Take $\varphi_{\gamma}=\delta_{t_{0}}$ to be a spike at location $t_{0} \in T^{\prime} \backslash T$. Using the uncertainty principle, we will show that $\delta_{t_{0}}$ cannot be in Range $\left(\Phi_{\Gamma}\right)$.

Arguing by contradiction, suppose that $\delta_{t_{0}} \in \operatorname{Range}\left(\Phi_{\Gamma}\right)$. Then there must be a linear combination of the sinusoids in $\Phi_{\Omega}$ that is zero everywhere except on $T \cup\left\{t_{0}\right\}$. Expressed differently, there exists $\alpha_{0}$ supported on $\Omega$ such that $f=F^{*} \alpha_{0}$ vanishes outside of $T \cup\left\{t_{0}\right\}$. Let $f_{T}$ be the values of $f$ on $T$, and $f_{\left\{t_{0}\right\}}$ the value at $t_{0}$. Since $\hat{f}$ is supported on $\Omega$ and the pair $(T, \Omega)$ obeys $(4.1)$, it follows that

$$
\left\|f_{T}\right\|_{2}^{2}=\left\|F_{\Omega T}^{*} R_{\Omega} \hat{f}\right\|^{2} \leq q\|f\|_{2}^{2}
$$

which gives $\left|f_{\left\{t_{0}\right\}}\right|^{2} \geq(1-q)\|f\|_{2}^{2}$. By construction, $1_{\Omega^{c}} \cdot \hat{f}=0$ or, equivalently, $F R_{T}^{*} f_{T}=f_{\left\{t_{0}\right\}} F \delta_{t_{0}}$ on $\Omega^{c}$ implying that

$$
\left\|1_{\Omega^{c}} \cdot F R_{T}^{*} f_{T}\right\|_{2}^{2}=\left|f_{\left\{t_{0}\right\}}\right|^{2}\left\|1_{\Omega^{c}} \cdot F \delta_{t_{0}}\right\|_{2}^{2}=\left|f_{\left\{t_{0}\right\}}\right|^{2} \cdot\left(1-\frac{|\Omega|}{N}\right) .
$$

On the one hand, we have

$$
\left\|1_{\Omega^{c}} \cdot F R_{T}^{*} f_{T}\right\|_{2}^{2} \leq\left\|f_{T}\right\|_{2}^{2} \leq q\|f\|_{2}^{2},
$$


and on the other,

$$
\begin{aligned}
\left|f_{\left\{t_{0}\right\}}\right|^{2}\left\|1_{\Omega^{c}} \cdot F \delta_{t^{\prime}}\right\|_{2}^{2} & \geq(1-q) \cdot\left(1-\frac{|\Omega|}{N}\right) \cdot\|f\|_{2}^{2} \\
& \geq(1-q) \cdot\left(1-\frac{.5583 q}{\sqrt{(\beta+1) \log N}}\right) \cdot\|f\|_{2}^{2} \\
& \geq(1-q) \cdot(1-.1581 q) \cdot\|f\|_{2}^{2},
\end{aligned}
$$

where the last inequality holds for $\beta \geq 1$ and $N \geq 512$. Therefore, (4.3) can hold only if

$$
q \geq(1-q) \cdot(1-.1581 q)
$$

which is not true for $q \leq .48026$. As a result, (4.2) holds, and $\alpha$ is $\ell_{0}$-unique with probability 1 (conditioned on $\Gamma$ obeying (4.1)).

The generalization to Theorem 5.2 (whose statement does not change) is also an easy change. We simply apply Theorem 5.1 with $C_{\beta}^{\prime}=C_{\beta} / 2$, using the same reasoning about support sizes as in Corollary 4.1.

\section{Acknowledgments}

The authors would like to thank Joel Tropp for pointing out the error. 